\title{
EFFECT OF BODY FORCES DUE TO AIRCRAFT ACCELERATION ON VENTILATION AIRFLOW AND CONTAMINANT DISPERSION IN PASSENGER AIRCRAFT CABINS
}

\author{
Hossam A. Elmaghraby \\ Mechanical Engineering Program, School of Engineering \\ University of Guelph \\ Guelph, Ontario N1G 2W1, Canada \\ helmaghr@uoguelph.ca
}

\author{
Yi Wai Chiang, Amir A. Aliabadi \\ Environmental Engineering Program, School of Engineering \\ University of Guelph \\ Guelph, Ontario N1G 2W1, Canada \\ chiange@uoguelph.ca \\ *Corresponding Author: aliabadi@uoguelph.ca
}

\begin{abstract}
Numerical simulations for the effect of body forces due to aircraft acceleration on the airflow and contaminant dispersion in a model for a passenger aircraft cabin are performed in this study. It was found that those body forces have a significant impact on the contaminant dispersion phenomena and concentrations, especially during the climb leg, where the concentration was almost triple its counterpart during the steady level flight case at the two monitoring locations. Air velocities, on the other side, increased noticeably during the climb and descent legs leading to evident changes in the airflow patterns, vorticity magnitudes, and at some locations, vorticity directions, as well.
\end{abstract}

Keywords-Aircraft ventilation; Air quality; Contaminants; Aircraft acceleration; Airflow vorticity; Numerical simulation; CFD

\section{INTRODUCTION}

Nowadays, billions of people every year prefer to use air travel rather than other means of transport because of the fast and reliable service the airliners provide. However, the environment inside a commercial aircraft cabin provides a fertile ground for deterioration of air quality and disease transmission among passengers if proper measures are not taken [1].

In the past two decades, numerous studies with different research approaches have investigated air quality in aircraft cabin environments using air distribution systems as a control measure. These approaches range from purely experimental [26] to entirely computational (numerical simulations) [7-9], or combinations of both [10-14].

Despite this large number of airflow and air quality studies in aircraft cabins, and although aircraft are moving with high speeds and accelerations accomplishing distinct flight mission legs (or intervals) with different dynamic conditions, such as takeoff, climb, cruise, descent, and landing [15], no study has considered the effect of the resulting body forces from these accelerations on the flow of ventilation air and contaminate dispersion inside aircraft cabins.
The main objective of this work is to fill the gap in the literature that always considered that the passenger aircraft are at rest or under cruise conditions, and that the ventilation airflow, buoyancy effects due to the occupants-generated thermal plumes, and contaminants dispersion within their cabins are only influenced by the gravitational acceleration. Moreover, the effect of the accelerations induced by the body forces acting on a typical modern passenger aircraft (Boeing 767-300), such as the lift and drag, in addition to the thrust of the jet engines and the weight of the aircraft itself, on the air distribution and gaseous contaminant dispersion inside an economy cabin section is to be investigated through computational fluid dynamics (CFD) simulations using the ANSYS FLUENT software. The mission legs, during which the simulations are performed. are climb, steady level cruise, and descent.

\section{METHODS}

\section{A. Simulated cabin geometry}

The aircraft cabin model used for the current study is adopted from the literature, and more specifically the experimental study in [4], and the numerical simulation followup work in [8].

The model resembles an actual size sectional economy-class cabin of a Boeing 767 passenger aircraft with 21 seats arranged in three rows (the seven abreast or 2-3-2 seat arrangement). The external dimensions of the cabin mockup are $4.9 \mathrm{~m}$ by $3.2 \mathrm{~m}$ by $2.1 \mathrm{~m}(\mathrm{~W}, \mathrm{~L}, \mathrm{H})$. The cabin mockup is located at the International Centre for Indoor Environment and Energy, Technical University of Denmark, Lyngby, Denmark. The cabin mockup appeared for the first time in the literature in [3] where more details about its configuration and control systems can be found. Fig. 1 shows a three-dimensional view for the created cabin model in the current study to the exact dimensions using the design software DesignModeler included in the commercial CFD package ANSYS 17.0, and a plan view for the seats.

\footnotetext{
*Address all correspondence to this author.
} 


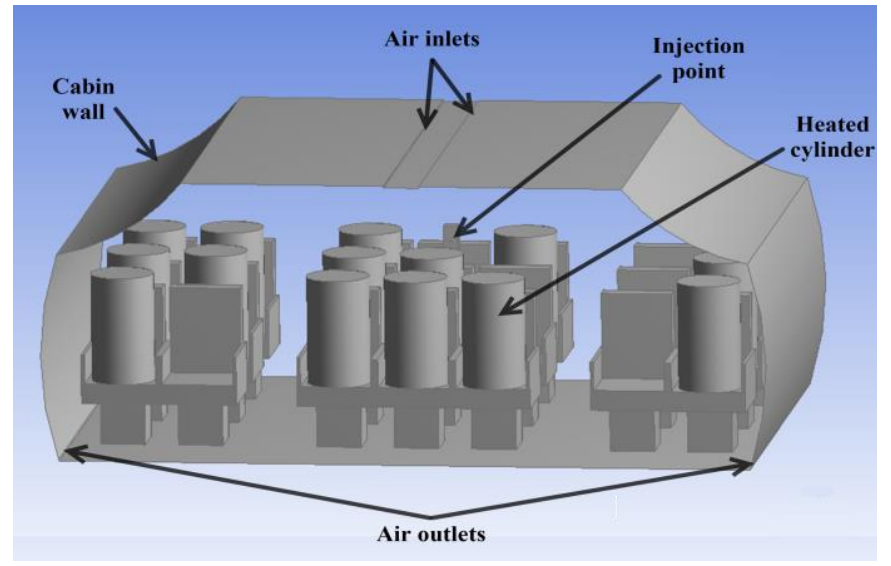

(a)

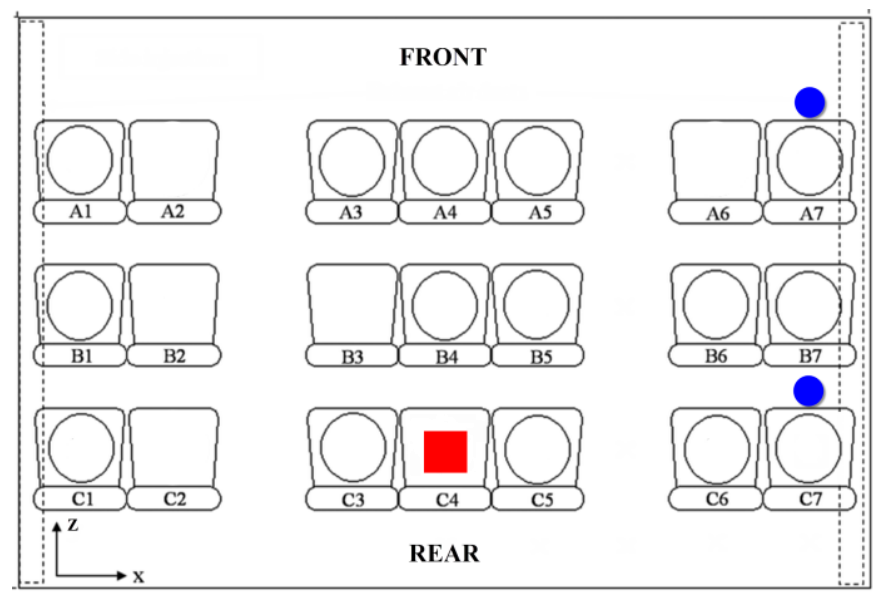

(b)

Figure 1. The computational cabin model used in the current study; (a) threedimensional view of the geometry built in ANSYS 17.0, and (b) plan view for the cabin with the cougher/injector position (red square), and the $\mathrm{SF}_{6}$ concentration monitoring points (blue circles)

\section{B. Model and boundary conditions}

The model's boundary conditions found in [4] and [8] were closely followed and implemented in the numerical solver FLUENT for the case of $200 \mathrm{~L} \mathrm{~s}^{-1}$ supply air flow rate, which was the only supply air flow rate considered. $\mathrm{SF}_{6}$ gas was released in the cabin to mimic the injection and transport of the cough's smallest size droplets (typically 1.6 to $3.0 \mu \mathrm{m}$ ), and which formed the largest number concentration of the injected droplet ensemble in the experiments. This was performed for the sake of model validation. Table I shows the boundary and inlet conditions for the current model.

The simulation time for the transient airflow part is $350 \mathrm{~s}$ at the start of which the cough $\left(\mathrm{SF}_{6}\right.$ release) was introduced for 1 $\mathrm{s}$ with released air volume of $0.4 \mathrm{l}$. Before this transient run, the airflow domain only was completely solved in the steady mode.
TABLE I. BOUNDARY AND INLET CONDITIONS FOR THE MODEL

\begin{tabular}{|l|l|}
\hline \multicolumn{1}{|c|}{ Boundary/inlet Conditions } & \multicolumn{1}{c|}{ Value } \\
\hline Supply air temperature & $24^{\circ} \mathrm{C}$ \\
\hline Supply air flow rate & $\begin{array}{l}200 \mathrm{~s}^{-1} \text { (corresponds to a supply } \\
\left.\text { velocity of } 2.61 \mathrm{~m} \mathrm{~s}^{-1}\right)\end{array}$ \\
\hline Supply air absolute humidity & $\begin{array}{l}0.92 \mathrm{~g} \mathrm{~kg}^{-1}(\text { corresponds to } 5 \% \mathrm{RH} \\
\text { at supply air temperature) }\end{array}$ \\
\hline Cabin wall temperature & $18{ }^{\circ} \mathrm{C}$ \\
\hline Heating cylinder heat release & $60 \mathrm{~W}$ per cylinder \\
\hline $\mathrm{SF}_{6}$ (cough) injection location & Seat $\mathrm{C} 4$ \\
\hline Air velocity at injection location & $10.6 \mathrm{~m} \mathrm{~s}^{-1}$ \\
\hline
\end{tabular}

The flow turbulence in the cabin was modeled using the renormalization group (RNG) k- $\varepsilon$ model of the Reynoldsaveraged Navier-Stokes (RANS) group based on recommendations for similar airflow simulation cases in closed spaces found in the literature [16-19].

\section{Grid independence test}

For defining the degree of independence of the obtained solution (airflow velocity and time-averaged $\mathrm{SF}_{6}$ concentration) from the grid size changes, a grid independence test is conducted. In the present work, three levels of grid fineness (sizes) were created, namely: coarse grid $(4,704,751$ elements), medium grid $(5,522,517$ elements), and fine grid $(7,375,800$ elements), in the order of grid element size decrease or fineness increase. The mesh refinement ratio ( $r$ ) between each two consecutive grid levels was kept constant at 1.1.

Transient simulation runs were performed for airflow and $\mathrm{SF}_{6}$ dispersion in the cabin on each grid level with the identical boundary conditions mentioned previously in section B. Fig. 2 presents the normalized $\mathrm{SF}_{6}$ concentration time series (real-time concentration divided by time-averaged concentration) as they change with the simulation time for the three grid levels with respect to the experimental data (see section $\mathrm{D}$ ). The real-time $\mathrm{SF}_{6}$ concentration was monitored at the breathing level of the occupants (1.17 m) at seats A7 and C7 (blue circles in Fig.1).

In addition to the graphical comparison of solution on the three grid levels, the grid convergence index (GCI) is calculated to indicate the amount of asymptotic convergence that the solution achieves through determining the uncertainty in solution between two consecutive grid levels [20,21].

In the current study, the GCI is calculated using the following formula:

$$
G C I_{m n}=\frac{F_{s}\left|\epsilon_{m n}\right|}{r^{p}-1}
$$

where, $F_{s}$ is a factor of safety recommended to be 3.0 for comparisons of two meshes and 1.25 for comparison of three meshes (such as in the current model), $\epsilon_{m n}$ is relative error between the two solutions obtained on two consecutive grid levels, and $p$ is the order of convergence. For more information on the calculation procedure followed refer to $[21,22]$. 


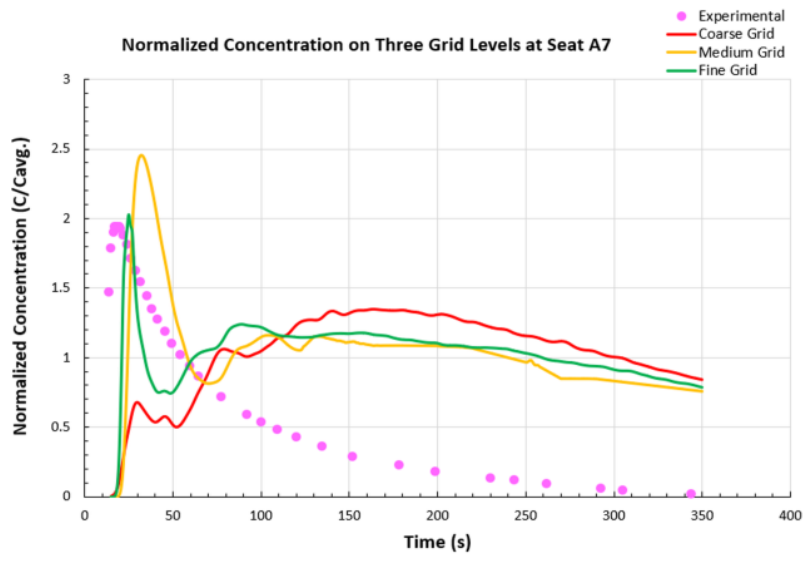

(a)

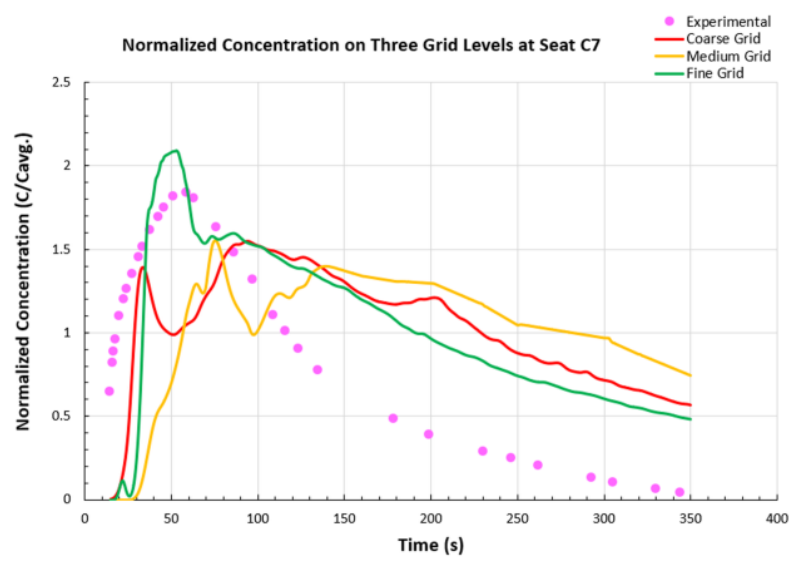

(b)

Figure 2. Normalized $\mathrm{SF}_{6}$ concentration change with the simulation time on the three grid levels; (a) at seat A7, and (b) at seat C7

The GCI for $\mathrm{SF}_{6}$ concentration calculations from the coarse to the medium grids was $3.13 \%$, and from the medium to the fine grids was $1.26 \%$ at seat A7. On the other hand, at seat C7, the GCI was $3.55 \%$ from the coarse to the medium grids, while it was equal to $1.41 \%$ between the medium and the fine grids. Based on these results, the fine grid level was found to exhibit enough grid independency of the solution, and therefore, will be used further.

\section{Model validation and error estimation}

The experimental data used for the aircraft cabin model validation was mainly extracted from the original study [4] considering the smallest particle size range $(1.6-3.0 \mu \mathrm{m})$ to be compared with the $\mathrm{SF}_{6}$ (passive tracer) concentration time series monitored at the two seats $\mathrm{A} 7$ and $\mathrm{C} 7$ over the simulation time. This is based on the findings of multiple studies in literature that indicate that the smallest size droplets ( 3 microns in diameter and less) behave like the gaseous substances (especially $\mathrm{SF}_{6}$ ) when dispersing in the cabin space $[5,10]$. Fig. 3 depicts the compared normalized concentration time series at seats $\mathrm{A} 7$ and $\mathrm{C} 7$ using the numerical solution obtained on the fine grid only.

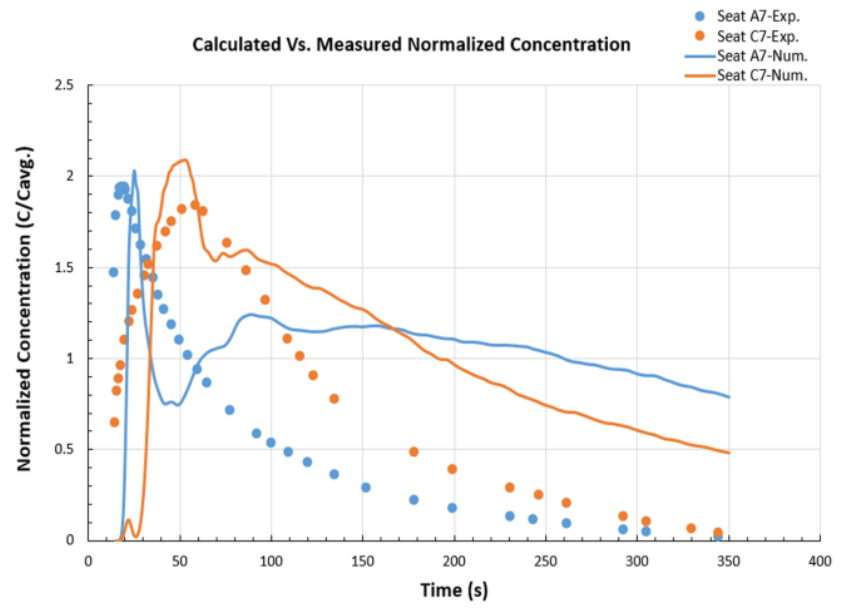

Figure 3. Comparison of the normalized concentration time series between the experiment and numerical simulation on the fine grid level

The error estimation in the current CFD model predictions for airflow velocity and concentration time series are calculated using a procedure which was first proposed by Steven Hanna in [23], and used by him and others later in multiple studies to express the error between the observed and predicted concentrations in atmospheric air quality models [24-26]. This procedure uses two performance measures to express the error, namely: the fractional mean bias (FB), and the normalized mean square error (NMSE), defined as follows:

$$
F B=\frac{2\left(\overline{C_{o}-C_{p}}\right)}{\left(\overline{C_{o}}+\overline{C_{p}}\right)},
$$

$$
N M S E=\frac{\left(\overline{\left(C_{o}-C_{p}\right)^{2}}\right)}{\left(\overline{C_{o}} * \overline{C_{p}}\right)},
$$

where, $C_{o}$ and $C_{p}$ are the observed and predicted concentrations, respectively.

Table II gives the FB and NMSE values calculated for the velocity magnitudes and normalized concentration time series between the experimental measurements and numerical predictions in the current study.

From Table II, it can be remarked that both error measures are noticeably lower for the air velocity magnitude than for the normalized concentration calculations. On the other hand, the NMSE values for normalized concentration at seat $\mathrm{C} 7$ are about $50 \%$ less than at seat A7, while FB values are almost the same at both seats. This shows that the shift between the observed and predicted concentration values is similar, but the spread of the predicted data with respect to the observed ones is two times higher at seat A7, which indicates less accurate predictions.

The decrease in prediction accuracy from seat $\mathrm{C} 7$ to seat $\mathrm{A} 7$ 
TABLE II.

FB AND NMSE VALUES FOR THE NUMERICAL PREDICTIONS

\begin{tabular}{|cc|cc|cc|}
\hline \multicolumn{2}{|c|}{ Air Velocity } & \multicolumn{4}{|c|}{ Normalized Concentration } \\
\hline \multirow{2}{*}{ FB } & NMSE & $\begin{array}{c}\text { FB } \\
\text { (Seat A7) }\end{array}$ & $\begin{array}{c}\text { NMSE } \\
\text { (Seat A7) }\end{array}$ & $\begin{array}{c}\text { FB } \\
\text { (Seat C7) }\end{array}$ & $\begin{array}{c}\text { NMSE } \\
\text { (Seat C7) }\end{array}$ \\
\hline 0.07977 & 0.02743 & 0.09705 & 0.96112 & 0.06922 & 0.42384 \\
\hline
\end{tabular}

can very well be attributed to the condition of airflow, and therefore that of the surrogate $\mathrm{SF}_{6}$ gas, from the emission source (at seat C4) to each seat. From C4 to C7 the flow is mainly lateral which is less susceptible to the bulk flow turbulence than the primarily longitudinal flow experienced from $\mathrm{C} 4$ to A7 (see Fig. 1).

Generally, the calculated FB and NMSE figures agree well with the graphical presentation for the measured and calculated concentration time series (Fig. 3).

\section{E. Calculation of aircraft body acceleration components}

The aircraft vertical acceleration $\left(a_{v}\right)$ and horizontal acceleration $\left(a_{h}\right)$ components were calculated during climb and descent legs using a basic approach adapted from different sources in aircraft dynamics literature [27] and online [28,29].

The calculation procedure relies on applying Newton's second law $\left(\sum \vec{F}=m \vec{a}\right)$ on two axes passing through the center of gravity of the aircraft; one is vertical and the other is horizontal. The forces in action are the lift (L) and drag (D) on the aircraft, in addition to the aircraft's weight (W) and the thrust of the jet engines (T). More information on the calculation procedure followed can be found in appendices A and $\mathrm{B}$ at the end of this paper.

\section{RESULTS AND DISCUSSION}

\section{A. Effect of aircraft body forces on contaminants dispersion}

The climb and descent simulations were run for the same time span of the steady level flight simulations (350 s), but with the new acceleration components, resulting from the climb and descent calculations, implemented in the numerical solver. Fig. 4 shows a comparison of the calculated concentration time series of $\mathrm{SF}_{6}$ during steady level flight, climb, and descent at the two set monitoring locations at seats A7 and C7.

It can be clearly noticed from Fig. 4 that the tracer gas concentration is significantly higher (up to $250 \%$ more) during the climb leg than the steady level flight case for most of the simulation time at the two locations. Consequently, the level of exposure of passengers sitting at any of the two seats to the contaminant, which can be interpreted from the area under the curves, significantly increases throughout the aircraft climb time. This poses greater infection risks on the health and wellbeing of most passengers in the cabin upon exposure to hazardous gaseous substances, in-cabin contaminants, or some infectious particulates during the aircraft climb leg that can take up from 20 to 30 minutes for some flights and aircraft models.

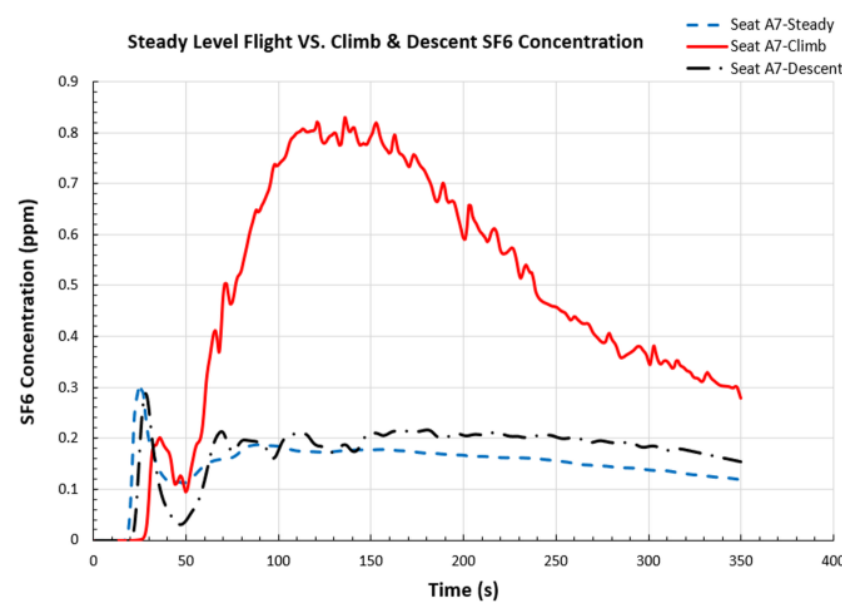

(a)

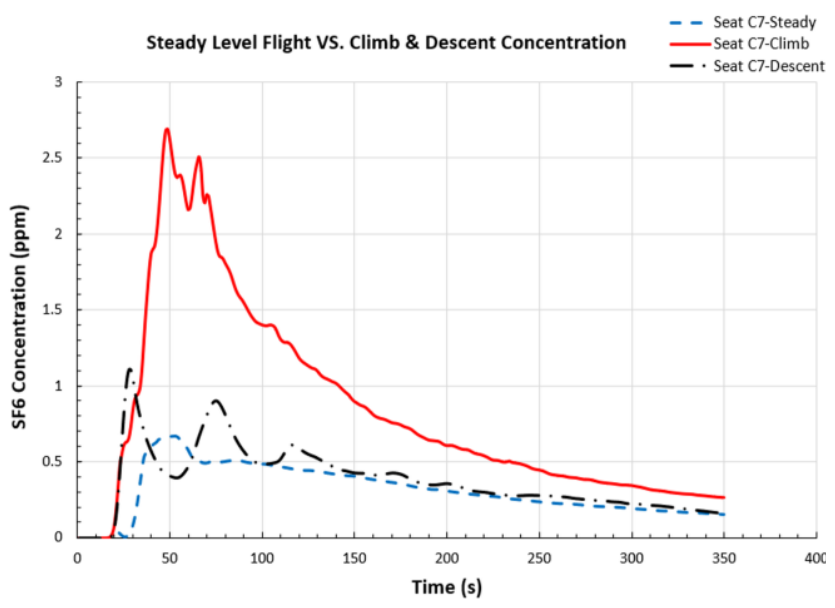

(b)

Figure 4. Comparison of the predicted $\mathrm{SF}_{6}$ concentration time series among steady level flight, climb, and descent scenarios; (a) at seat A7, and (b) at seat C7

On the other hand, the $\mathrm{SF}_{6}$ concentration time series during the descent leg does not noticeably differ from that for the steady level flight time, and the former can be seen increasing slightly over the latter at the beginning or near the end of the simulation run depending on the monitoring point location. This indicates that the level of passengers' exposure to contaminants is almost identical between the descent and the steady level flight scenarios at the breathing height. However, such similarity in the exposure between the two cases can be altered by changing the contaminant injection point or direction and/or varying the number of concentration monitoring sites and their locations, such factors is beyond the scope of the current study.

One factor that may have contributed to the considerable difference in the $\mathrm{SF}_{6}$ concentration time series between the climb and the descent legs is the difference between climb and descent rate of velocity change and the corresponding accelerations. The climb speed for most modern jet-powered passenger aircraft can reach up to $600 \mathrm{~km} \mathrm{hr}^{-1}$ (324 knots) or more, while the full-powered descent rate is limited to around 
$250 \mathrm{~km} \mathrm{hr}^{-1}$ (135 knots) only to ensure passengers' comfort. This large difference in aircraft speed between the two mission legs yield dissimilar aircraft accelerations, and therefore, distinct effect of the generated body forces on the contaminants dispersion rates and patterns inside the cabin.

Another factor in play is the difference between the climb and the descent (inclination) angles. During the take-off and climb legs, most jet-powered passenger aircraft adopt an inclination angle from $15^{\circ}$ to $20^{\circ}$, such an angle is much steeper than the small descent angle restricted mostly to $3^{\circ}$ to perform comfortable descents. As much as the rise in aircraft speed, the increase of the climb angle over the descent angle very well contribute in enhancing the dispersion rate of contaminants and changing their distribution contours within the cabin. This is because changing the flight path angles significantly alters the values of body force components acting on the aircraft in motion and the contained air on the vertical and horizontal lines, and therefore, the aircraft acceleration components in each of those two directions.

\section{B. Effect of aircraft body forces on airflow patterns and vorticity}

In addition to the effect the body forces have on the contaminant dispersion inside the cabin, they also affect the airflow velocity and patterns, which can be quantified using vorticities.

Airflow velocity magnitudes in all directions inside the cabin were in general greater during climb and descent than that throughout steady level flight. The monitored airflow velocity magnitudes for the simulated flight time increased anywhere between $1 \%$ and $45 \%$ during climb and between $6 \%$ and $42 \%$ during descent with respect to the steady level flight air velocity magnitudes. On the other hand, the air velocity components $\left(\mathrm{V}_{\mathrm{x}}\right.$, $\mathrm{V}_{\mathrm{y}}$, and $\mathrm{V}_{\mathrm{z}}$ ) exhibited different values of increase and decrease between climb and steady flight, and descent and steady flight, with no fixed trend.

The increase in airflow velocity magnitudes throughout the climb and descent legs changed the airflow patterns in the cabin to some degree. One form of this change is the increased air boundary-layer thickness adjacent to the cabin walls due to the tendency of the air to separate from the walls as it moves downward during climb and descent. In other words, the increased downward airflow velocities $\left(0.9 \mathrm{~m} \mathrm{~s}^{-1}\right.$ for climb and $0.8 \mathrm{~m} \mathrm{~s}^{-1}$ for descent compared to $0.65 \mathrm{~m} \mathrm{~s}^{-1}$ during the steady level flight) led to reduce the airflow attachment to the walls. Consequently, the strongest downward flow separation (thickest airflow boundary-layer) is noticed during climb.

Another effect the airflow velocity changes have on airflow patterns is the alteration of the vorticity magnitude and direction in the cabin. For rotational (non-zero vorticity) flows, such as the highly turbulent airflow in the cabin space, the vorticity vector $(\vec{\omega})$ is defined as the curl of the velocity as follows:

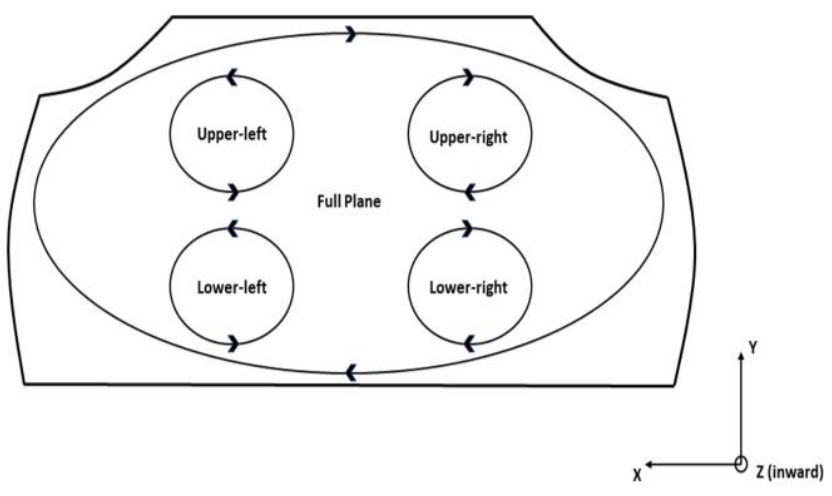

Figure 5. Example of the followed notation for estimating the z-component airflow vorticity $\left(\omega_{z}\right)$ on the full $x-y$ plane and its four side corners

$$
\vec{\omega}=\nabla \times \vec{V}=\omega_{x} \hat{\imath}+\omega_{y} \hat{\jmath}+\omega_{z} \hat{k}
$$

Fig. 5 shows an example of the airflow vorticity on the $x-y$ plane set at the middle of the cabin. From the figure, it can be clearly seen that the airflow vorticity is used to approximate circulation on the full planes, such as $x-y, x-z$, and $z-y$ (not shown in the figure, but corresponding to $\omega_{z}, \omega_{y}$, and $\omega_{x}$, respectively), and also at the four side corners of each plane, namely: the upper-left, upper-right, lower-left, and lower-right corners. This was made possible by calculating the average airflow velocities on separate line segments located at the top, bottom, right, left, and the center (horizontally and vertically) of each of the mentioned planes. Afterwards, the difference in magnitude between each pair of those velocities, and the distances between each two parallel lines on which they were calculated, are used to yield the vorticity vector components as follows:

$$
\begin{aligned}
& \omega_{x}=\frac{\Delta v_{z}}{\Delta y}-\frac{\Delta v_{y}}{\Delta z} \\
& \omega_{y}=\frac{\Delta v_{x}}{\Delta z}-\frac{\Delta v_{z}}{\Delta x} \\
& \omega_{z}=\frac{\Delta v_{y}}{\Delta x}-\frac{\Delta v_{x}}{\Delta y}
\end{aligned}
$$

Table III presents the calculated vorticity vector magnitudes and components on the full planes and the four side corners of each during the steady level flight, climb, and descent legs, and also, the percentage change of those values when each of the climb and descent legs is compared to the steady level flight case.

Studying Table III, it can be noticed that the vorticity vector magnitudes during climb and descent are always greater than those during steady level flight either on the full plane or any of 
TABLE III. AIRFLOW VORTICITY VALUES AND CHANGES BETWEEN STEADY LEVEL FLIGHT, CLIMB AND DESCENT LEGS

\begin{tabular}{|c|c|c|c|c|c|c|c|c|c|}
\hline \multirow{2}{*}{ Plane/side corner } & \multirow{2}{*}{ Flight Leg } & \multirow{2}{*}{$\begin{array}{c}\text { Vorticity }^{\mathrm{a}} \\
\text { Magnitude }\end{array}$} & \multicolumn{3}{|c|}{ Vorticity Components ${ }^{\mathrm{b}}$} & \multicolumn{4}{|c|}{ Change in Vorticity During Climb/Descent ${ }^{\mathrm{c}}(\%)$} \\
\hline & & & $\omega_{x}$ & $\omega_{y}$ & $\omega_{z}$ & $\begin{array}{c}\text { Vorticity } \\
\text { Magnitude }\end{array}$ & $\omega_{x}$ & $\omega_{y}$ & $\omega_{z}$ \\
\hline \multirow{3}{*}{ Full Plane } & Steady Flight & 0.009 & -0.004 & -0.0065 & 0.0046 & - & - & - & - \\
\hline & Climb & 0.014 & -0.01 & -0.0105 & 0.002 & 63.98 & 158.1 & 62.05 & -64.64 \\
\hline & Descent & 0.023 & 0.012 & -0.019 & 0.0043 & 163.6 & $221.1^{d}$ & 197.7 & -6.45 \\
\hline \multirow{3}{*}{ Lower-right Corner } & Steady Flight & 0.168 & 0.028 & -0.023 & 0.164 & - & - & - & - \\
\hline & Climb & 0.196 & -0.033 & -0.015 & 0.193 & 16.85 & $19.64^{\mathrm{d}}$ & -34.13 & 17.59 \\
\hline & Descent & 0.194 & 0.072 & -0.028 & 0.178 & 15.49 & 161.3 & 17.71 & 8.53 \\
\hline \multirow{3}{*}{ Lower-left Corner } & Steady Flight & 0.141 & 0.023 & -0.007 & -0.139 & - & - & - & - \\
\hline & Climb & 0.200 & 0.079 & -0.024 & -0.182 & 42.07 & 249.2 & 243.9 & 31.12 \\
\hline & Descent & 0.154 & -0.021 & -0.002 & -0.152 & 9.050 & $-9.83^{\mathrm{d}}$ & -70.06 & 9.64 \\
\hline \multirow{3}{*}{ Upper-right corner } & Steady Flight & 0.151 & -0.030 & -0.006 & 0.148 & - & - & - & - \\
\hline & Climb & 0.210 & -0.099 & 0.003 & 0.185 & 39.28 & 226.6 & $-57.81^{d}$ & 25.42 \\
\hline & Descent & 0.170 & 0.045 & -0.036 & 0.160 & 13.01 & $47.49^{d}$ & 498.3 & 8.64 \\
\hline \multirow{3}{*}{ Upper-left corner } & Steady Flight & 0.159 & -0.035 & 0.0105 & -0.155 & - & - & - & - \\
\hline & Climb & 0.190 & 0.013 & -0.006 & -0.189 & 19.33 & $-61.66^{d}$ & $-47.39^{d}$ & 22.25 \\
\hline & Descent & 0.177 & -0.048 & -0.0109 & -0.169 & 10.82 & 35.94 & $3.99^{\mathrm{d}}$ & 9.41 \\
\hline
\end{tabular}

a. The unit for vorticity magnitude and components is $\mathrm{s}^{-1}$

b. (+) is in clockwise direction and (-) is in counterclockwise direction

c. (+) indicates an increase and (-) indicates a decrease from steady flight

d. Accompanied with a change in the direction of rotation

the four corners. This rise is mainly caused by the increased airflow velocity gradients everywhere in the cabin during climb and descent. This indicates stronger circulation of air in the cabin for each zone identified. Between climb and descent, the former has higher vorticity magnitudes at the corners, while this was not the case on the full plane only, where circulation patterns on each corner cancel each other out on the full plane. The greatest increase in vorticity magnitude, taking the steady level flight case as a reference, is seen on the full plane during descent with around $164 \%$ of increase. This was followed by the climb on the full plane, as well, with $64 \%$ increase, while, for the corners, the amounts of increase were relatively close in value and in the favor of climb, as previously mentioned.

On the other hand, the vorticity vector components exhibited both increases and decreases during climb and descent, with respect to the steady level flight case, with increases occurring slightly more frequently. However, those increases are not exclusive to one flight leg, and occur almost equally between climb and descent. One unique characteristic of vorticity components is that they can show the change in the vorticity direction between steady flight and climb, and steady flight and descent, in addition to the increase or decrease in magnitude. This is shown by a change in the sign of the vorticity component from positive (clockwise direction) to negative (counterclockwise direction), and vice versa. Those cases are also clearly demonstrated in Table 3 tagged by the footnote (d) to show that a change in the direction of airflow rotation occurs even if the vorticity intensity (magnitude) increases or decreases.

\section{CONCLUSIONS}

In this study, numerical simulations for the effect of body forces due to aircraft acceleration on the airflow and contaminant dispersion in a model for a passenger aircraft cabin are performed. The steady level flight leg which takes most of the flight time is taken as the reference case, to which the contaminant concentration and airflow changes during the climb and descent flight legs are compared. 
It was noticed that the concentration of the contaminant surrogate $\left(\mathrm{SF}_{6}\right)$ at the two set monitoring locations, and therefore the passengers' exposure, increased substantially during the climb leg from the steady level flight. However, this was not the case during the descent leg, throughout which the $\mathrm{SF}_{6}$ concentration did not considerably differ from that during the steady level flight time.

Airflow velocity magnitudes, on the other hand, increased everywhere in the simulated cabin during climb and descent from the steady flight case. But, this was not the case for the airflow velocity components $\left(\mathrm{V}_{\mathrm{x}}, \mathrm{V}_{\mathrm{y}}\right.$, and $\left.\mathrm{V}_{\mathrm{z}}\right)$ which showed different levels of increase and decrease with no fixed trend.

The change in airflow velocities had a significant effect on the airflow patterns and vorticity (approximation for circulation) in the cabin. Downward airflow coming from supply slots was less attached to the cabin walls during climb and descent than through the steady flight time. Additionally, airflow vorticity magnitudes always exhibited an increasing trend when the steady flight case was changed to either the climb or descent scenario, indicating greater air circulation in the cabin. This increasing trend, however, was not followed by the vorticity components on the full plane and its corners in each direction which showed various percentages of increase and decrease, and also displayed changes in the vorticity direction from steady flight to either climb or descent.

The findings indicate the potent effect the body forces have on the airflow behavior and contaminate dispersion inside the cabins of passenger aircraft and calls for more research attention to this topic to unveil some ventilation design remedies to the negative effects this may have on the health of aircraft occupants.

\section{ACKNOWLEDGMENT}

The authors thank the Government of Ontario, Canada, for providing the funding for this work in the form of the Ontario Trillium Scholarship (OTS) for the lead author. They also thank Christopher Y. H. Chao (Department of Mechanical Engineering, Hong Kong University of Science and Technology, Clear Water Bay, Hong Kong, China), and Man Pun Wan (Department of Mechanical \& Aerospace Engineering, Nanyang Technological University, Singapore) for providing their full research data. Finally, the authors recognize CMC Microsystems for providing the Academic Research license of ANSYS, and the Centre for Advanced Computing (CAC) at Queen's University (Kingston, ON, Canada) for providing the Frontenac Compute Cluster to run the simulations.

\section{REFERENCES}

[1] H. A. Elmaghraby, Y. W. Chiang, and A. A. Aliabadi, "Ventilation strategies and air quality management in passenger aircraft cabins: A review of experimental approaches and numerical simulations," Sci. Technol. Built Environ., vol. 24, no. 2, pp. 160-175, 2018.

[2] M. A. Waters, T. F. Bloom, B. Grajewski, and J. Deddens, "Measurements of Indoor Air Quality on Commercial Transport Aircraft," in The 9th International Conference on Indoor Air
Quality and Climate, 2002, pp. 782-787.

[3] P. Strøm-Tejsen, D. P. Wyon, L. Lagercrantz, and L. Fang, "Passenger evaluation of the optimum balance between fresh air supply and humidity from 7-h exposures in a simulated aircraft cabin," Indoor Air, vol. 17, no. 2, pp. 92-108, 2007.

G. N. Sze To, M. P. Wan, C. Y. H. Chao, L. Fang, and A. Melikov, "Experimental Study of Dispersion and Deposition of Expiratory Aerosols in Aircraft Cabins and Impact on Infectious Disease Transmission," Aerosol Sci. Technol., vol. 43, no. 5, pp. 466-485, 2009.

[5] F. Li, J. Liu, J. Pei, C. H. Lin, and Q. Chen, "Experimental study of gaseous and particulate contaminants distribution in an aircraft cabin," Atmos. Environ., vol. 85, pp. 223-233, 2014.

[6] Z. Fang, H. Liu, B. Li, A. Baldwin, J. Wang, and K. Xia, "Experimental investigation of personal air supply nozzle use in aircraft cabins," Appl. Ergon., vol. 47, pp. 193-202, 2015.

C.-H.- Lin, K. H. Dunn, R. H. Horstman, J. L. Topmiller, M. F. Ahlers, J. S. Bennett, L. M. Sedgwick, and S. Wirogo, "Numerical Simulation of Airflow and Airborne Pathogen Transport in Aircraft Cabins--Part I: Numerical Simulation of the Flow Field.," ASHRAE Trans., vol. 111, no. 1, pp. 755-764, 2005.

M. P. Wan, G. N. Sze To, C. Y. H. Chao, L. Fang, and A. Melikov, "Modeling the Fate of Expiratory Aerosols and the Associated Infection Risk in an Aircraft Cabin Environment," Aerosol Sci. Technol., vol. 43, no. 4, pp. 322-343, 2009.

[9] M. Hassan, "Numerical Investigation of Improving Distribution Systems In Aircraft Passengers Cabins," Cairo University, Giza, Egypt, 2016.

[10] Z. Zhang, X. Chen, S. Mazumdar, T. Zhang, and Q. Chen, "Experimental and numerical investigation of airflow and contaminant transport in an airliner cabin mockup," Build. Environ., vol. 44, no. 1, pp. 85-94, 2009.

[11] S. B. Poussou, S. Mazumdar, M. W. Plesniak, P. E. Sojka, and Q. Chen, "Flow and contaminant transport in an airliner cabin induced by a moving body: Model experiments and CFD predictions," Atmos. Environ., vol. 44, no. 24, pp. 2830-2839, 2010.

[12] T. T. Zhang, P. Li, and S. Wang, "A personal air distribution system with air terminals embedded in chair armrests on commercial airplanes," Build. Environ., vol. 47, no. 1, pp. 89-99, 2012.

[13] S. S. Isukapalli, S. Mazumdar, P. George, B. Wei, B. Jones, and C. P. Weisel, "Computational fluid dynamics modeling of transport and deposition of pesticides in an aircraft cabin," Atmos. Environ., vol. 68, pp. 198-207, 2013.

[14] F. Li, J. Liu, J. Ren, X. Cao, and Y. Zhu, "Numerical investigation of airborne contaminant transport under different vortex structures in the aircraft cabin," Int. J. Heat Mass Transf., vol. 96, pp. 287295, 2016.

[15] D. G. Hull, Fundamentals of Airplane Flight Mechanics. New York: Springer Berlin Heidelberg, 2007.

[16] Z. J. Zhai, Z. Zhang, W. Zhang, and Q. (Yan) Chen, "Evaluation of Various Turbulence Models in Predicting Airflow and Turbulence in Enclosed Environments by CFD: Part 1-Summary of Prevalent Turbulence Models," HVAC\&R Res., vol. 13, no. 6, pp. 853-870, 2007.

[17] Z. Zhang, W. Zhang, Z. J. Zhai, and Q. Y. Chen, "Evaluation of Various Turbulence Models in Predicting Airflow and Turbulence in Enclosed Environments by CFD: Part 2-Comparison with Experimental Data from Literature," $H V A C \& R$ Res., vol. 13, no. 6, pp. 871-886, 2007. 
[18] M. Wang and Q. Chen, "Assessment of various turbulence models for transitional flows in enclosed environment (RP-1271)," HVAC\&R Res., vol. 15, no. 6, pp. 1099-1119, 2009.

[19] W. Liu, J. Wen, C. H. Lin, J. Liu, Z. Long, and Q. Chen, "Evaluation of various categories of turbulence models for predicting air distribution in an airliner cabin," Build. Environ., vol. 65, pp. 118-131, 2013.

[20] P. J. Roache, "Perspective: A Method for Uniform Reporting of Grid Refinement Studies," J. Fluids Eng., vol. 116, no. 3, pp. 405413, 1994.

[21] A. A. Aliabadi, Theory and Applications of Turbulence: A Fundamental Approach for Scientists and Engineers. Guelph, ON, Canada: Amir Abbas Aliabadi Publications, 2018.

[22] A. A. Aliabadi, "Dispersion of Expiratory Airborne Droplets in a Model Single Patient Hospital Recovery Room with Stratified Ventilation," The University of British Columbia, Vancouver, Canada, 2013.

[23] S. R. Hanna, "Confidence limits for air quality model evaluations, as estimated by bootstrap and jackknife resampling methods," Atmos. Environ., vol. 23, no. 6, pp. 1385-1398, 1989.

[24] J. C. Chang and S. R. Hanna, "Air quality model performance evaluation," Meteorol. Atmos. Phys., vol. 87, no. 1-3, pp. 167196, 2004.

[25] S. Hanna and J. Chang, "Acceptance criteria for urban dispersion model evaluation," Meteorol. Atmos. Phys., vol. 116, no. 3-4, pp. 133-146, 2012.

[26] A. A. Aliabadi, E. S. Krayenhoff, N. Nazarian, L. W. Chew, P. R Armstrong, A. Afshari, and L. K. Norford, "Effects of Roof-Edge Roughness on Air Temperature and Pollutant Concentration in Urban Canyons," Boundary-Layer Meteorol, vol. 164, no. 2, pp. 249-279, 2017.

[27] S. Gudmundsson, General Aviation Aircraft Design: Applied Methods and Procedures. Butterworth-Heinemann, 2013.

[28] National Aeronautics and Space Adminstration (NASA), "Forces in a climb," 2015. [Online]. Available: https://www.grc.nasa.gov/www/k-12/airplane/climb.html\%0A. [Accessed: 10-Oct-2017].

[29] University of Southampton, "Weight, Geometry, Lift, Drag and Thrust Properties," 2005. [Online]. Available: http://www.southampton.ac.uk/ jps7/Aircraft Design Resources/Sydney aerodynamics for students/perf/perf_ac.html. [Accessed: 10-May-2017]. 


\section{Appendix (A): Procedure FOr Determining THE AIRCRAFT ACCELERATION COMPONENTS DURING CLIMB}

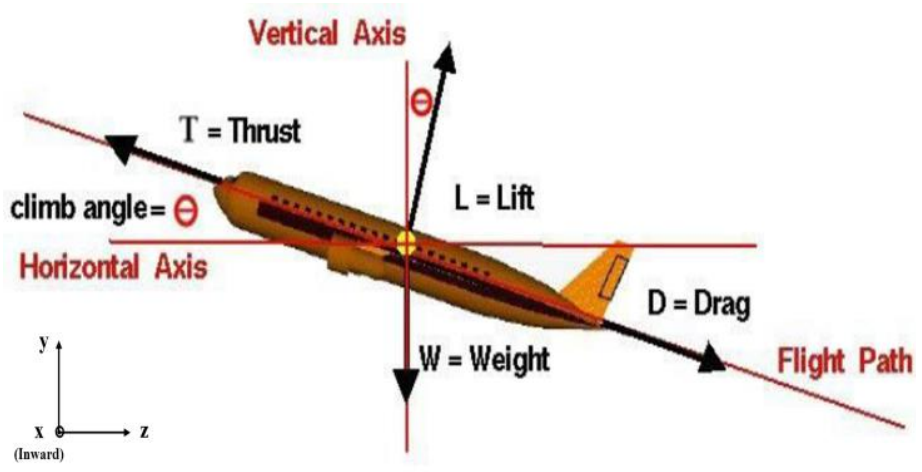

Figure 6. Forces on a passenger aircraft during climb with the two axes (horizontal and vertical) set for the calculation of acceleration components

Newton's second law is applied on the vertical and horizontal axes shown in the above figure,

$$
\sum \vec{F}=m \vec{a}
$$

On the vertical axis:

$$
\mathrm{T} \sin \theta-\mathrm{D} \sin \theta+\mathrm{L} \cos \theta-\mathrm{W}=\mathrm{m} \overrightarrow{\mathrm{a}_{\mathrm{v}}},
$$

and, on the horizontal axis:

$$
\mathrm{T} \cos \theta-\mathrm{D} \cos \theta+\mathrm{L} \sin \theta=\mathrm{m} \overrightarrow{\mathrm{a}_{\mathrm{h}}},
$$

where $\overrightarrow{\mathrm{a}_{\mathrm{v}}}$ and $\overrightarrow{\mathrm{a}_{\mathrm{h}}}$ are the vertical and horizontal acceleration components, respectively.

The unknowns $(\mathrm{T}, \theta, \mathrm{D}, \mathrm{L}, \mathrm{m})$ are estimated based on industrial specifications and dimensions for the Boeing 767300 aircraft.

$T=462.6 \mathrm{KN}$ (for a twin-jet engine)

$\boldsymbol{\theta}=\mathbf{2 0}{ }^{\circ}$

$\mathbf{m}=\mathbf{1 5 9 , 2 1 0} \mathrm{kg}$ (max. takeoff weight)

$\mathrm{W}=\boldsymbol{m g}$

$$
\begin{aligned}
& \mathrm{D}=\mathrm{C}_{\mathrm{D}} * 0.5 \rho \mathrm{V}^{2} \mathrm{~A}, \\
& \mathrm{~L}=\mathrm{C}_{\mathrm{L}} * 0.5 \rho \mathrm{V}^{2} \mathrm{~A},
\end{aligned}
$$

where $\mathrm{C}_{\mathrm{D}}$ and $\mathrm{C}_{\mathrm{L}}$ are the drag and lift coefficients, respectively, $\rho$ is the air density, $V$ is the aircraft velocity (taken as $\mathbf{1 5 5} \mathrm{m} \mathrm{s}^{-1}$ ), and $A$ is the reference (wing) area.

$$
\mathrm{C}_{\mathrm{D}}=\mathrm{C}_{\mathrm{D}_{0}}+\mathrm{kC}_{\mathrm{L}}^{2}
$$

where $C_{D_{0}}$ is the part of drag coefficient due to friction and pressure on the aircraft body, and $k$ is a constant that incorporates the other part of the drag coefficient due to lift (lift induced drag).

$\mathbf{C}_{\mathbf{D}}=\mathbf{0 . 0 6}$ (approximation for the majority of aircraft aerofoils)

$$
\mathrm{k}=\frac{1}{\pi \mathrm{ARe}}
$$

where $e$ is a constant equal to 0.85 for twin engine wide-body aircraft, and $A R$ is the wing aspect ratio which is determined from:

$$
\mathrm{AR}=\frac{(\text { wing span })^{2}}{\text { wing area }}=\frac{(47.57)^{2}}{283.3}=7.987
$$

These yield $\mathbf{k}=\mathbf{0 . 0 4 6 8 8}$

Taking $C_{D_{0}}=0.017$ for twin engine wide-body, and substituting in equation 6 , yields $\mathbf{C}_{\mathbf{L}}=\mathbf{0 . 9 6}$

Substituting in equations 4 and 5, and assuming the density of air to be $1.2 \mathrm{~kg} \mathrm{~m}^{-3}$ (aircraft still close to sea level), the drag and lift forces on the aircraft during climb are determined to be:

$$
\mathrm{D}=229.473 \mathrm{KN} \text {, }
$$

and

$$
\mathrm{L}=3671.568 \mathrm{KN} \text {. }
$$

Substituting in equations 2 and 3 yields the vertical and horizontal components of the aircraft acceleration, respectively:

and

$$
\overrightarrow{\mathrm{a}_{\mathrm{v}}}=13.79 \mathrm{~ms}^{-2}=-1.4 \vec{g},
$$

$$
\overrightarrow{\mathbf{a}_{\mathrm{h}}}=-7.14 \mathrm{~ms}^{-2}=0.73 \vec{g} \text {. }
$$

Lastly, the absolute acceleration components got are expressed in the form of relative acceleration components on the air inside the aircraft cabin before being implemented in Fluent. This is attained by reversing the sign of each acceleration component and superimposing it on any acceleration(s) that may exist in the same direction (e.g. gravity). This yield the relative acceleration components as follows:

$$
\begin{gathered}
\overrightarrow{\mathrm{a}_{\mathrm{y}}}=(-13.79-9.81)=-23.6 \mathrm{~ms}^{-2}=2.4 \vec{g}, \\
\left(\text { or } 23.6 \mathrm{~ms}^{-2} \text { acting downwards }\right)
\end{gathered}
$$

and

$$
\overrightarrow{\mathrm{a}_{\mathrm{z}}}=7.14 \mathrm{~ms}^{-2}=-0.73 \vec{g} \text {. }
$$

(or $7.14 \mathrm{~ms}^{-2}$ acting toward the tail of the aircraft) 


\section{ApPendix (B): Procedure FOR DETERMining THE AIRCRAFT ACCELERATION COMPONENTS DURING DESCENT}

For descent, the same procedure and parameters for climb apply, but the aircraft velocity $(\mathrm{V})$, descent angle $(\theta)$, and the density of air $(\rho)$, at the chosen descent elevation $(10000 \mathrm{ft}$. or $3050 \mathrm{~m}$ ), are adjusted to the new case.

$\mathbf{V}=\mathbf{7 0} \mathbf{m ~ s}^{-1}$ (full-powered descent)

$\boldsymbol{\theta}=\mathbf{3}^{\circ}$

$\rho=0.9 \mathrm{~kg} \mathrm{~m}^{-3}$

With the aircraft tilted downward, equations 2 and 3 in Appendix (A) are changed to:

On the vertical axis:

$$
\mathrm{L} \cos \theta+\mathrm{D} \sin \theta-\mathrm{T} \sin \theta-\mathrm{W}=\mathrm{m} \overrightarrow{\mathrm{a}_{\mathrm{v}}} \text {, }
$$

and, on the horizontal axis:

$$
\mathrm{L} \sin \theta+\mathrm{T} \cos \theta-\mathrm{D} \cos \theta=\mathrm{m} \overrightarrow{\mathrm{a}_{\mathrm{h}}}
$$

Substituting in equations 4 and 5 of Appendix (A) with the new velocity and air density, and using the same values for $\mathrm{C}_{\mathrm{D}}$ and $\mathrm{C}_{\mathrm{L}}$, yields:

and

$$
\mathrm{D}=37.481 \mathrm{KN}
$$

$$
\mathrm{L}=599.69 \mathrm{KN} \text {. }
$$

Substituting the D and $\mathrm{L}$ values got (keeping $\mathrm{T}$ and $\mathrm{m}$ the same) in equations 2 and 3 of Appendix (A) yields the vertical and horizontal components of aircraft acceleration during descent, respectively:

and

$$
\overrightarrow{a_{v}}=-6.1883 \mathrm{~ms}^{-2}=0.63 \vec{g}
$$

$$
\overrightarrow{a_{h}}=2.86 \mathrm{~ms}^{-2}=-0.29 \vec{g}
$$

Finally, the relative acceleration components are calculated for the descent leg using the same approach previously followed for the climb leg:

$$
\begin{gathered}
\overrightarrow{\mathrm{a}_{\mathrm{y}}}=(6.1883-9.81)=-3.622 \mathrm{~ms}^{-2}=0.37 \vec{g}, \\
\left(\text { or } 3.622 \mathrm{~ms}^{-2} \text { acting downwards }\right)
\end{gathered}
$$

and

$$
\overrightarrow{\mathrm{a}_{\mathrm{z}}}=-2.86 \mathrm{~ms}^{-2}=0.29 \overrightarrow{\mathrm{g}} \text {. }
$$

(or $2.86 \mathrm{~ms}^{-2}$ acting toward the head of the aircraft) 